\title{
LINC00511 promotes proliferation and invasion by sponging miR-515-5p in gastric cancer
}

Di Wang ${ }^{1}$, Ketong Liu² and Erdong Chen ${ }^{1 *}$

* Correspondence:
erdongcheneast@163.com
1Department of Gastroenterology I,
The Third Affiliated Hospital of
Qiqihar Medical University, Qiqihar
161000, Heilongjiang, China
Full list of author information is
available at the end of the article

\begin{abstract}
Background: Long non-coding RNAs (IncRNAs) are known to be involved in tumorigenesis. The functions of LINC00511 in gastric cancer are poorly understood.

Methods: Quantitative RT-PCR was performed to investigate the levels of LINC00511 in gastric cancer tissues and cell lines. CCK-8, flow cytometry, wound-healing and Transwell assays were performed to examine cellular functions. The underlying regulatory mechanisms of LINC00511 in gastric cancer progression were determined using luciferase reporter and RIP assays.

Results: LINC00511 levels were significantly higher in gastric cancer tissues and cell lines than in normal samples. The high expression of LINC00511 in gastric cancer patient samples positively correlated with advanced clinical characters and poor prognosis. Depleting LINC00511 reduced tumor cell proliferation, migration and invasion, slowed tumor growth, and accelerated cell apoptosis. Our mechanistic study results indicated that LINC00511 promotes gastric cancer progression in a miR515-5p-dependent manner.

Conclusion: We established that LINC00511 may contribute to the proliferation and invasion of gastric cancer cells by modulating miR-515-5p, indicating that LINC00511 may be a potential molecular target for the development of anti-cancer drugs.
\end{abstract}

Keywords: Gastric cancer, LINC00511, miR-515-5p, ceRNA

\section{Introduction}

Gastric cancer is the fifth most common cancer and the third major cause of cancerrelated mortality worldwide $[1,2]$. Current treatment approaches consist of a combination of surgery, radiation and chemotherapy. Although treatment advancements have been made, the prognosis remains unsatisfactory due to metastasis and chemoresistance $[3,4]$. It is vital to understand the molecular mechanisms for the growth and metastasis of gastric cancer and identify effective treatment approaches.

Long non-coding RNAs (lncRNAs) are RNA molecules that are longer than $200 \mathrm{nu}$ cleotides and incapable of being translated into proteins [5]. Various reports have shown that lncRNAs participate in growth, metastasis, differentiation and apoptosis, among other processes [6, 7]. Zhou et al. showed that a decrease in the levels of the lncRNA LET correlated with a worse prognosis in gastric cancer patients [8]. Li et al. suggested that CASC2 suppressed gastric cancer cell proliferation through the MAPK

(C) The Author(s). 2020 Open Access This article is distributed under the terms of the Creative Commons Attribution 4.0 International License (http://creativecommons.org/licenses/by/4.0/), which permits unrestricted use, distribution, and reproduction in any medium, provided you give appropriate credit to the original author(s) and the source, provide a link to the Creative Commons license, and indicate if changes were made. The Creative Commons Public Domain Dedication waiver (http://creativecommons.org/ publicdomain/zero/1.0/) applies to the data made available in this article, unless otherwise stated. 
pathway [9]. Liu et al. revealed that GAS5 acted as a molecular sponge, regulating miR23a expression in gastric cancer [10].

We explored the function of the lncRNA LINC00511 on the development and metastasis of gastric cancer and the underlying mechanisms. Our results reveal for the first time that LINC00511 promotes the growth and invasion of gastric cancer cells through sponging miR-515-5p. This discovery provides a possible new treatment target for this malignant tumor.

\section{Materials and methods}

Patients and specimens

The subjects were 25 patients who had been diagnosed with gastric cancer (age range, 36-81; 14 male patients and 11 female patients) and were due to undergo surgery in The Third Affiliated Hospital of Qiqihar Medical University. None of the patients received radiotherapy or chemotherapy prior to the operation. During the operation, tumor and corresponding healthy gastric tissue samples were collected and immediately snap frozen... The experimental protocols were approved by the Research Ethics Committee of The Third Affiliated Hospital of Qiqihar Medical University.

\section{Cell culture and transfection}

A human gastric epithelial cell line (GES-1) and five human gastric cancer cell lines (AGS, SGC7901, BGC823, MKN45, MGC803) were obtained from then American Type Culture Collection (ATCC). All cells were grown in Dulbecco's modified Eagle's medium (DMEM; Invitrogen), which was augmented with $10 \%$ fetal bovine serum (FBS; Invitrogen), and cultured at $37^{\circ} \mathrm{C}$ in a humidified atmosphere containing $5 \% \mathrm{CO}_{2}$.

Short hairpin RNA targeting LINC00511 (sh-LINC00511) [11], miR-515-5p mimics, miR-515-5p inhibitors and scramble controls were customized by GenePharma and applied to co-transfected target cells per the manufacturer's instructions.

\section{RNA extraction and quantitative RT-PCR analyses}

TRIzol (Invitrogen) reagent was used to isolate $1 \mu \mathrm{g}$ of total RNA and then reversetranscribed into cDNA using PrimeScript RT Reagent Kit (Takara) according to the manufacturer's instructions. Next, the SYBR Premix Ex Taq II kit (Takara) and the ABI 7500HT Real-Time PCR System (Applied Biosystems) were used to perform real-time PCR. Expression of RNA was normalized to either U6 or GAPDH and calculated using the CT method $\left(2^{-\triangle \triangle C T}\right)$. The primer sequences were: LINC00511, forward 5' - CTAA CAAGAGGGTAAGTGTCAG-3' and reverse 5'-AAGTCGACAACCCCATCGTTAC -3' [11]; miR-515-5p, forward 5'-TTCTCCAAAAGAAAGCACTTTCTG-3' and reverse 5'-CTCGCTTCGGCAGCACA-3' [12]; GAPDH, forward 5'-CATCAAGAAG GTGGTGAAGCAG-3' and reverse 5' -CGTCAAAGGTGGAGGAGTGG-3'; and U6, forward 5' -GCGCGTCGTGAAGCGTTC-3' and reverse 5' -GTGCAGGGTCCGAGG-3' .

\section{GEPIA and Kaplan-Meier plotter databases}

GEPIA data was used to obtain LINC00511 expression levels in stomach adenocarcinoma tissues and healthy tissues. The prognosis of gastric cancer patients was explored using the online Kaplan-Meier Plotter database. 


\section{CCK-8 assay}

After transfection, the cells were seeded onto 96 -well plates $\left(2 \times 10^{3}\right.$ cells/well $)$ as indicated for 24, 48, 72 and $96 \mathrm{~h}$. Next, $10 \mu \mathrm{l}$ of the solutions from the Cell Counting Kit-8 (CCK-8; Roche) were added to every well and incubated for $2 \mathrm{~h}$. Lastly, the optical density (OD) value of each well was quantified at $450 \mathrm{~nm}$ wavelength using a microplate reader (Bio- $\mathrm{Rad})$.

\section{Colony formation assay}

The colony formation assay was performed using the method detailed in a previous report [12].

\section{Flow cytometry assays}

Cell apoptosis was determined using a BD Biosciences cell apoptosis kit per the manufacturer's instructions. FACS flow cytometry (Beckman) was used to analyze apoptosis. It was also applied for the cell-cycle analysis, using the method detailed in a previous report [10].

\section{Wound-healing assay}

Transfected cells $\left(1 \times 10^{5}\right.$ cells/well $)$ were seeded and plated onto 6-well plates until they reached confluence. Next, a homogeneous wound was generated in the monolayer using a sterile plastic micropipette tip. The wound closure was imaged after 0 and $24 \mathrm{~h}$ using an Olympus microscope.

\section{Transwell invasion assay}

The top inserts of the Transwell chambers were coated with $50 \mu \mathrm{l}$ of Matrigel (BD Biosciences) and left overnight at $4{ }^{\circ} \mathrm{C}$. Then, $200 \mu \mathrm{l}$ of cell suspension that contained $1 \times$ $10^{5}$ transfected cells in serum-free medium was placed into the top inserts. After $48 \mathrm{~h}$, the cells were fixed and stained with $0.1 \%$ crystal violet. The quantity of cells that had passed through the Matrigel were measured using an Olympus microscope.

\section{In vivo tumorigenesis}

Animal experiments were approved by the Animal Care and Use Committee at Qiqihar Medical University. BALB/c nude mice that were 4-6 weeks old and weighed 18-20 g were obtained from the Laboratory Animal Research Centre at Qiqihar Medical University. $5 \times 10^{6}$ cells transfected with either sh-LINC00511 or sh-NC were subcutaneously inoculated in the mice's right flanks. The width (W) and length (L) of the xenografts that formed were quantified every 3 days. The tumor volume was estimated based on the formula

$$
\text { Volume }(\mathrm{V})=0.5 \times \mathrm{L} \times \mathrm{W}^{2}
$$

After 21 days, the mice were killed via cervical dislocation and the xenografts were removed and weighed.

\section{Western blotting}

Western blotting was conducted as described previously [9]. The primary antibodies included: ERK1/2 (cat. no. ab205718; 1:1000; Abcam), JNK (cat. no. ab131499; 1:1000; 
Abcam), p38 MAPK (cat. no. ab47363; 1:1000; Abcam), and GAPDH (1:10000; cat. no. sc-32,233; Santa Cruz Biotechnology). HRP-linked secondary antibodies were also brought from Santa Cruz Biotechnology. The levels of protein were revealed using ECL reagents (Amersham Biosciences).

\section{Immunohistochemical staining}

Paraffin-embedded tissue sections were heated at $60^{\circ} \mathrm{C}$ for $2 \mathrm{~h}$, dewaxed with xylene and hydrated sequentially with gradient ethanol. The activity of endogenous peroxidase was inhibited using 3\% hydrogen peroxide, and non-specific sites were blocked using serum. After antigen retrieval, the tissue sections were incubated with Ki-67 antibody (1: 500; Abcam) overnight at $4{ }^{\circ} \mathrm{C}$. After washing, the tissue sections were incubated with homologous secondary antibody for $2 \mathrm{~h}$ at room temperature. Finally, the tissue sections were stained with a $3,3^{\prime}$-diaminobenzidine solution and hematoxylin.

\section{Luciferase reporter assay}

BGC823 cells $\left(3 \times 10^{4}\right.$ cells/well $)$ were planted into 24 -well plates for $24 \mathrm{~h}$ and cotransfected with LINC00511-WT or LINC00511-Mut with or without miR-515-5p mimics using Lipofectamine 2000 reagents (Invitrogen). After $24 \mathrm{~h}$, the cells were collected and luciferase activity was quantified using the Dual-Luciferase Reporter Assay System (Promega) according to the manufacturer's instructions. Firefly luciferase activity was divided by Renilla luciferase activity for every sample to determine the transfection efficiency, which was used to normalize the data.

\section{RIP assay}

An RNA immunoprecipitation (RIP) assay was performed as described previously [13].

\section{Statistical analysis}

SPSS 16.0 software was used to conduct the statistical analysis. The results were evaluated as the means \pm SD of at least 3 independent experiments. Differences between two groups were compared through an independent t-test. Multiple groups were compared using one-way ANOVA. $p<0.05$ indicated statistical significance.

\section{Results}

\section{LINC00511 was upregulated in gastric cancer}

LINC00511 expression was determined to examine its function in gastric cancer progression. The GEPIA database revealed that LINC00511 is upregulated in stomach adenocarcinoma tissues and correlates with advanced tumor stage (Fig. 1a and b). Kaplan-Meier analysis showed that high LINC00511 levels predicted poor disease-free survival in gastric cancer patients (Fig. 1c).

We also evaluated LINC00511 expression in 25 paired gastric cancer tissues. LINC00511 expression was obviously higher in gastric cancer tissues (Fig. 1d). High LINC00511 expression levels correlated with bigger tumor size and advanced TNM stage in gastric cancer patients (Fig. 1e and f). Those results indicate that LINC00511 is involved in gastric cancer tumorigenesis. 


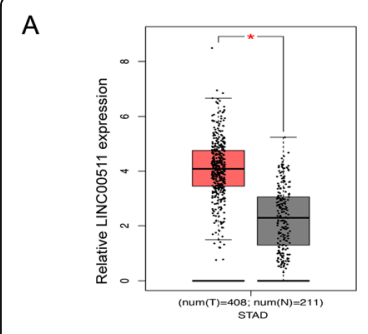

D

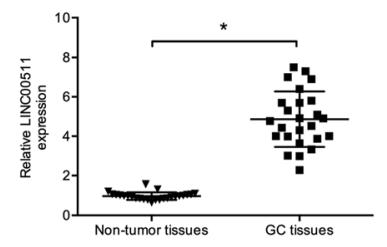

B

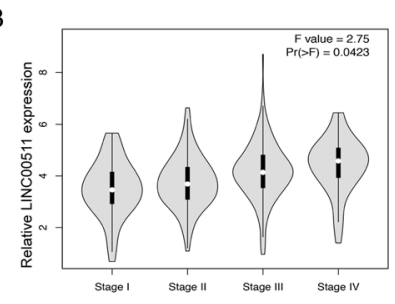

E

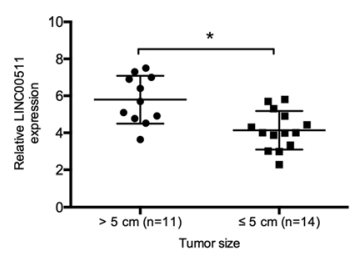

C

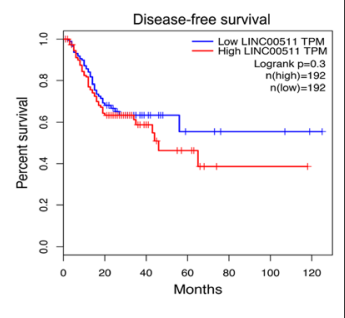

F

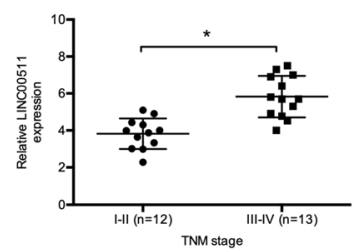

Fig. 1 The LnCRNA LINC00511 is upregulated in gastric cancer. a and $\mathbf{b}$ The relative LINC00511 expression in gastric cancer tissues, based on the TCGA dataset. c Disease-free survival analysis based on the TCGA dataset. d LINC00511 expression in 25 paired gastric cancer tissues was evaluated using quantitative RT-PCR. e and $\mathbf{f}$ High levels of LINC00511 correlated with larger tumor size and advanced TNM stage in GC patients. ${ }^{*} p<0.05$. Individuals were separated into two groups based on median gene expression

\section{Knockdown of LINC00511 inhibited gastric cancer progression}

The functions of LINC00511 in gastric cancer progression were explored. Quantitative RT-PCR revealed that levels of LINC00511 were significantly higher in cells of the gastric cancer lines AGS, SGC7901, BGC823, MKN45 and MGC803 than in those of the human gastric epithelial cell line GES-1 (Fig. 2a). After sh-LINC00511 and sh-NC vectors were transfected into BGC823 cells (Fig. 2b), the CCK-8 assay showed LINC00511 knockdown significantly blocked their proliferative and colony formation ability (Fig. 2c and d). The flow cytometry assay revealed that LINC00511 knockdown increased apoptosis in BGC823 cells and arrested them in G0/G1 phase (Fig. 2e and f). Furthermore, the Transwell assay showed that LINC00511 inhibition decreased the invasive ability of BGC823 cells (Fig. 2g and h).

\section{Knockdown of LINC00511 reduced the activation of the MAPK signaling pathway}

The MAPK signaling pathway involving JNK, ERK and p38-MAPK, which play critical roles in tumor progression [14]. We explored the impact of LINC00511 on MAPKrelated gene expression in BGC823 cells. Quantitative RT-PCR showed p38, ERK1/2 and JNK RNA levels were significantly lower in BGC823 cells transfected with shLINC00511 than in the sh-NC group (Fig. 3a). This is consistent with the protein expression levels of these genes (Fig. 3b). These findings support an LINC00511 contribution to gastric cancer progression through the MAPK signaling pathway.

\section{MiR-515-5p is a target of LINC00511}

A previous report stated that lncRNAs could serve as a sponge for modulating the expression and activity of miRNA [15]. The target prediction tool DIANA was used to assess putative miRNAs that interact with LINC00511. It demonstrated that LINC00511 possesses a putative miR-515-5p-binding site (Fig. 4a). Quantitative RT-PCR data 


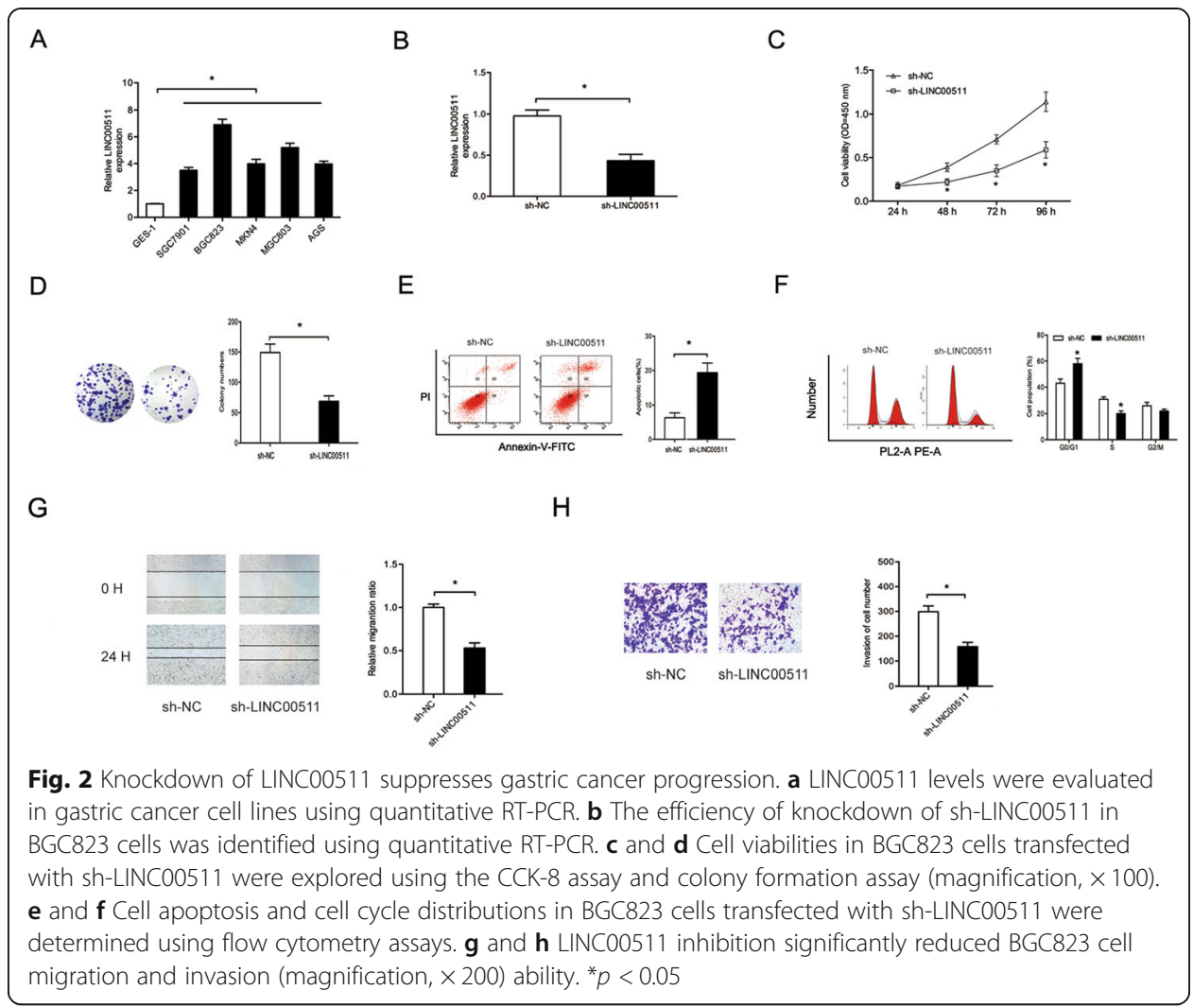

demonstrated that miR-515-5p levels were significantly lower and associated with poor overall survival in gastric cancer patients (Fig. 4c).

Next, we showed that miR-515-5p overexpression significantly inhibited the luciferase activity of the LINC00511-WT group in BGC823 cells (Fig. 4d). LINC00511 knockdown also significantly upregulated miR-515-5p in BGC823 cells (Fig. 4e). The RIP assay results indicated that both LINC00511 and miR-515-5p were enriched in the mixture immunoprecipitated by anti-Ago2 (Fig. 4f). Moreover, the expression of LINC00511 negatively correlated with miR-515-5p in gastric cancer tissues (Fig. 4g). Therefore, LINC00511 might interact with miR-515-5p in gastric cancer progression.

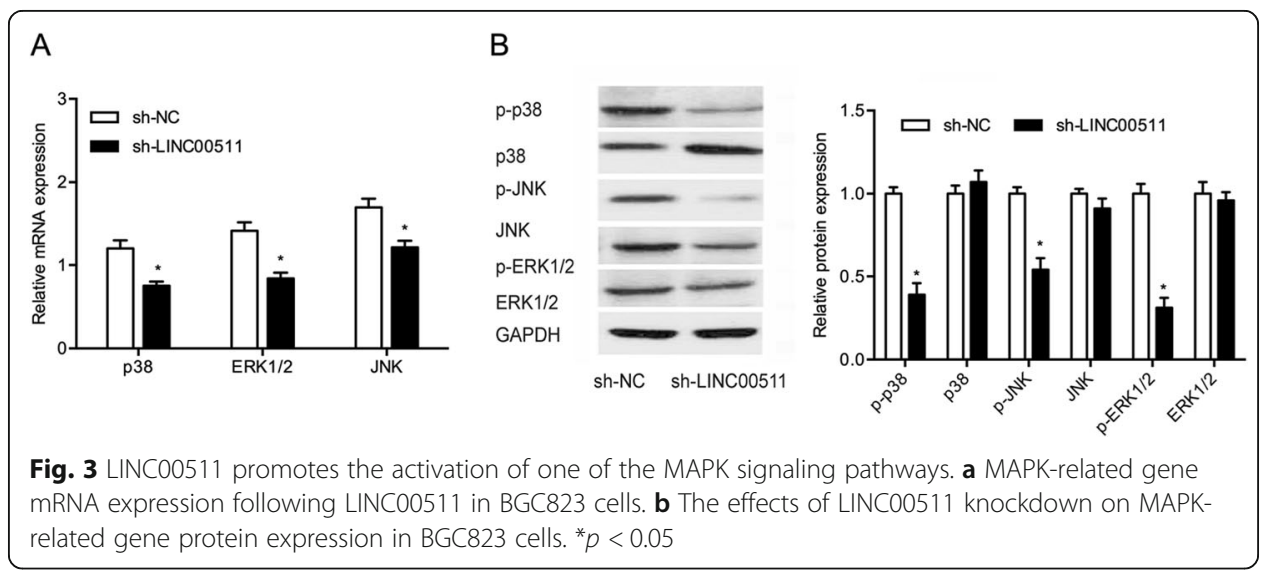




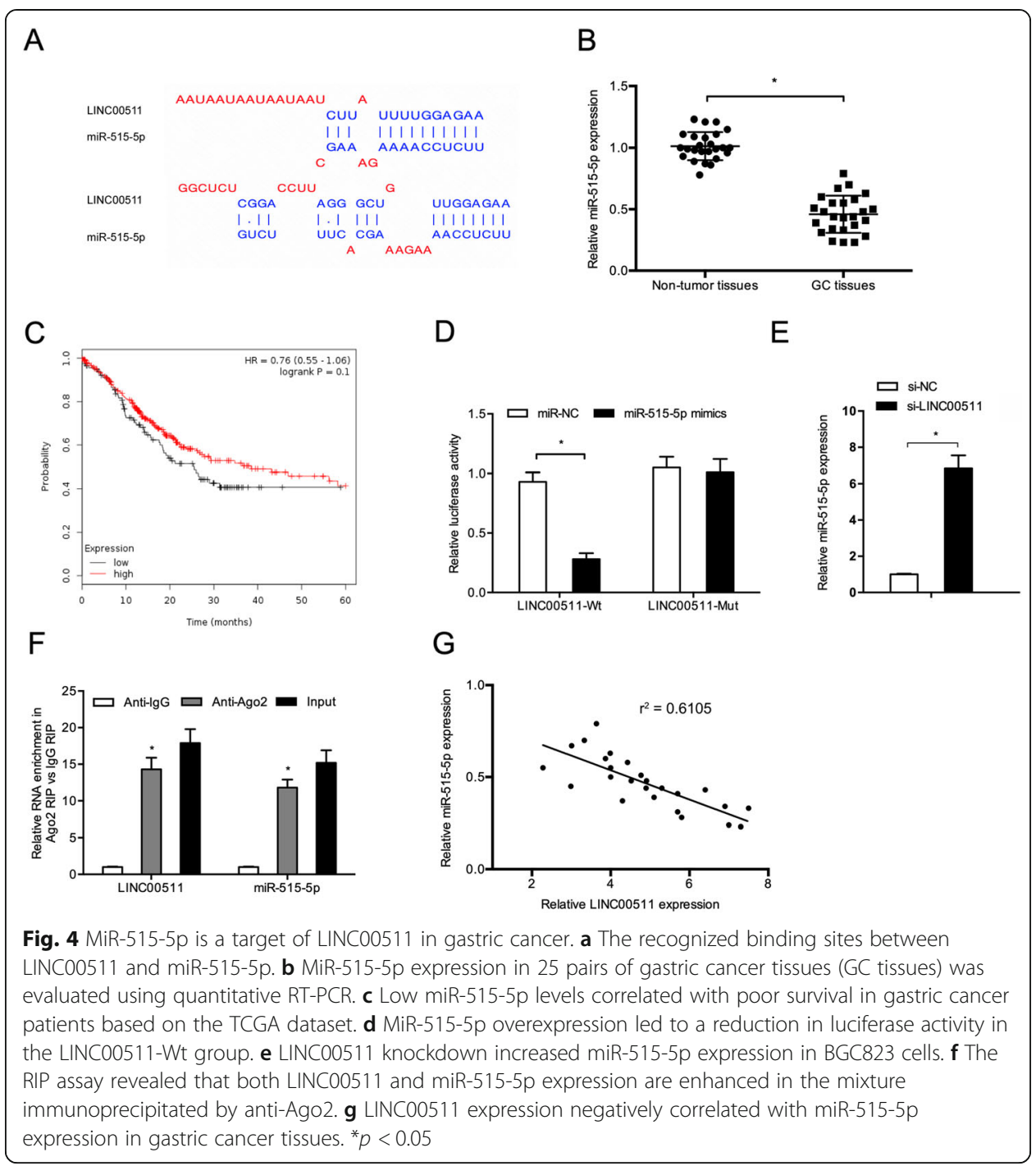

\section{LINC00511 knockdown inhibited tumor growth in vivo}

We further explored the roles of LINC00511 in tumor growth in vivo. Knockdown of LINC00511 significantly reduced growth of the xenograft of gastric cancer cells (Fig. 5a). Xenograft tumor weights and volumes were also significantly reduced by LINC00511 knockdown (Fig. 5b and c). Furthermore, immunohistochemistry results showed that Ki-67 levels were significantly lower in the sh-LINC0051 group than in the sh-NC group (Fig. 5d). These data revealed that LINC00511 knockdown reduced gastric cancer tumorigenesis in vivo.

\section{Discussion}

Several lncRNAs have recently been identified as having functions in the development and progression of cancers. For example, Liang et al. showed that PTAR promoted ovarian cancer progression by regulating the miR-101-3p/ZEB1 axis [16]. Yang et al. found that HOXA11-AS promoted renal cancer cell proliferation and invasion by targeting the miR-146b-5p/MMP16 axis [17]. Gao et al. found that the MAGI1-IT1/miR- 


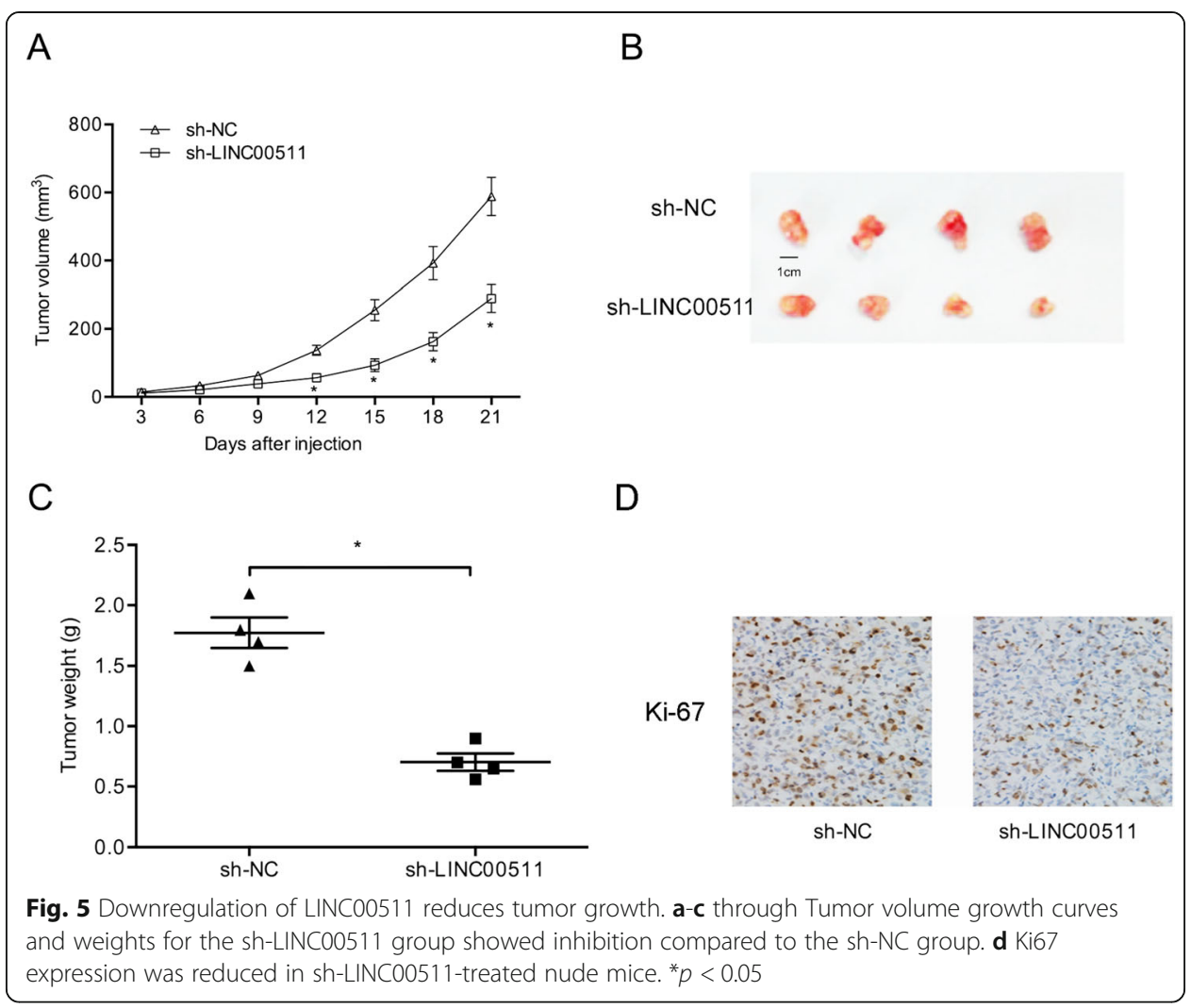

200a/ZEB axis could promote ovarian cancer cell metastasis [18]. However, the roles and underlying mechanisms of lncRNA in tumor progression remain unclear.

LINC00511 $(2.265 \mathrm{~kb})$ is a newly discovered IncRNA that is located on chromosome 17q24.3. It is reported to have critical functions in tumorigenesis. For example, Zhao et al. demonstrated that LINC00511 may be a ceRNA that controls VEGFA expression by sponging miR-29b-3p in pancreatic ductal adenocarcinoma [11]. Sun et al. found that LINC00511 promotes lung cancer progression by binding to EZH2 and inhibiting the expression of p57 [19]. Lu et al. showed that the LINC00511/miR-185-3p/E2F1/ Nanog axis might play critical roles in breast cancer tumorigenesis and maintenance of stemness [20]. Its function in gastric cancer remains unclear.

We found that LINC00511 was highly expressed in gastric cancer and that this expression positively correlated with larger tumor size, advanced TNM stage and poor disease-free survival. In function assays, LINC00511 knockdown inhibited proliferation, migration and invasion, and reduced tumor growth in vivo. LINC00511 knockdown significantly increased apoptosis and arrested the cell cycle in G0/G1 phase in BGC823 cells. These findings suggest that LINC00511 might act as an oncogenic lncRNA in gastric cancer tumorigenesis.

MAPK signaling pathways have a function in numerous cellular activities, including differentiation, proliferation, metastasis and drug resistance [21, 22]. For example, Song et al. found that the lncRNA ENST00000539653 promoted papillary thyroid cancer progression by regulating one of the MAPK signaling pathways [23]. Yang et al. reported that the lncRNA HOXD-AS1 suppressed proliferation and invasion in colorectal carcinoma by inhibiting the activation of integrin $\beta 3$ and the MAPK/AKT axis [24]. Liu 
et al. showed that the NEAT1/let-7a-5p/ Rsf-1 axis regulated nasopharyngeal carcinoma cell cisplatin resistance by regulating the activation of the Ras-MAPK pathway [25]. In our study, the expressions of p-p38, p-ERK and p-JNK were reduced by LINC00511 knockdown in BGC823 cells, suggesting the MAPK signaling pathway promotes the effect of LINC00511 on gastric cancer progression.

Studies have revealed that lncRNAs may act as sponges of miRNAs, further arresting the expression of mRNA [26]. Chen et al. demonstrated that the TTN-AS1/miR-573/ E2F3 axis plays critical roles in cervical cancer cell growth and metastasis [27]. Gao et al. revealed that suppressing LUCAT1 reduced glioma cell viability and invasion by modulating miR-375 expression [28]. Previous studies showed that miR-515-5p has critical roles in the progression of many tumors, including prostate cancer [29], lung cancer [30] and breast cancer [12]. However, the function of miR-515-5p in gastric cancer progression remains unclear. In this study, a DIANA analysis showed that LINC00511 possesses a putative miR-515-5p-binding site with a high score. LINC00511 knockdown increased the levels of miR-515-5p in BGC823 cells. Subsequently, overexpression of miR-515-5p significantly downregulated the luciferase activity of the LINC00511-Wt group. The correlation between LINC00511 and miR-515-5p was further confirmed using the RIP assay. In addition, miR-515-5p levels were significantly downregulated and negatively associated with the expression of LINC00511 in gastric cancer tissues. Therefore, we believe that LINC00511 encourages gastric cancer cell proliferation and invasion by sponging miR-515-5p.

\section{Conclusion}

We found that knockdown of LINC00511 could inhibit gastric cancer tumorigenesis through its sponging action on miR-515-5p. Our findings indicate that LINC00511 may be a novel therapeutic target for gastric cancer treatment.

Abbreviations

GC: Gastric cancer; LncRNA: Long non-coding RNAs; miRNAs: MicroRNAs; Mut: Mutant; UTR: Untranslated region; WT: Wild type

Acknowledgments

Not applicable.

Authors' contributions

DW and EDC conceived and designed this study. DW, KTL and EDC conducted the experiments and analyzed and checked the data. EDC supervised the whole project and revised the manuscript. All the authors read and approved the final manuscript.

Funding

Not applicable.

Availability of data and materials

The datasets supporting the conclusions of this article are included within the article.

Ethics approval and consent to participate

This research was approved by the Third Hospital Affiliated of Qiqihar Medical University and conducted in accordance with the principles of the Declaration of Helsinki (Number: THP-2018-B-002013).

Consent for publication

Not applicable.

Competing interests

The authors declare that they have no competing interests. 
Author details

1Department of Gastroenterology I, The Third Affiliated Hospital of Qiqihar Medical University, Qiqihar 161000, Heilongjiang, China. ${ }^{2}$ Department of Cardiology III, The Third Affiliated Hospital of Qiqihar Medical University, Qiqihar 161000, Heilongjiang, China.

Received: 20 June 2019 Accepted: 24 January 2020

Published online: 03 February 2020

References

1. Siegel RL, Miller KD, Jemal A. Cancer statistics, 2019. CA Cancer J Clin. 2019;69(1):7-34

2. Crew KD, Neugut Al. Epidemiology of gastric cancer. World J Gastroenterol. 2006;12(3):354.

3. Sitarz R, Skierucha M, Mielko J, Offerhaus GJA, Maciejewski R, Polkowski WP. Gastric cancer: epidemiology, prevention, classification, and treatment. Cancer Manag Res. 2018:10:239-48.

4. Rugge M, Genta RM, Di Mario F, El-Omar EM, El-Serag HB, Fassan M, et al. Gastric Cancer as preventable disease. Clin Gastroenterol Hepatol. 2017;15(12):1833-43.

5. Mattick JS, Makunin IV. Non-coding RNA. Hum Mol Genet. 2006;15 Spec No 1:R17-29.

6. Mercer TR, Dinger ME, Mattick JS. Long non-coding RNAs: insights into functions. Nat Rev Genet. 2009;10(3):155-9.

7. Fatica A, Bozzoni I. Long non-coding RNAs: new players in cell differentiation and development. Nat Rev Genet. 2014; 15(1):7-21.

8. Zhou B, Jing XY, Wu JQ, Xi HF, Lu GJ. Down-regulation of long non-coding RNA LET is associated with poor prognosis in gastric cancer. Int J Clin Exp Pathol. 2014;7(12):8893-8.

9. Li P, Xue WJ, Feng Y, Mao QS. Long non-coding RNA CASC2 suppresses the proliferation of gastric cancer cells by regulating the MAPK signaling pathway. Am J Transl Res. 2016;8(8):3522-9.

10. Liu X, Jiao T, Wang Y, Su W, Tang Z, Han C. Long non-coding RNA GAS5 acts as a molecular sponge to regulate miR23a in gastric cancer. Minerva Med. 2016;24:S232

11. Zhao X, Liu Y, Li Z, Zheng S, Wang Z, Li W, et al. Linc00511 acts as a competing endogenous RNA to regulate VEGFA expression through sponging hsa-miR-29b-3p in pancreatic ductal adenocarcinoma. J Cell Mol Med. 2018;22(1):655-67.

12. Pardo OE, Castellano L, Munro CE, Hu Y, Mauri F, Krell J, et al. miR-515-5p controls cancer cell migration through MARK4 regulation. EMBO Rep. 2016;17(4):570-84.

13. Li X, Yan X, Wang F, Yang Q, Luo X, Kong J, et al. Down-regulated IncRNA SLC25A5-AS1 facilitates cell growth and inhibits apoptosis via miR-19a-3p/PTEN/PI3K/AKT signaling pathway in gastric cancer. J Cell Mol Med. 2019;23(4):2920-32.

14. Johnson GL, Lapadat R. Mitogen-activated protein kinase pathways mediated by ERK, JNK, and p38 protein kinases. Science. 2002:298(5600):1911-2.

15. Zhang HM, Yang FQ, Chen SJ, Che J, Zheng JH. Upregulation of long non-coding RNA MALAT1 correlates with tumor progression and poor prognosis in clear cell renal cell carcinoma. Tumour Biol. 2015;36(4):2947-55.

16. Liang H, Yu T, Han Y, Jiang H, Wang C, You T, et al. LncRNA PTAR promotes EMT and invasion-metastasis in serous ovarian cancer by competitively binding miR-101-3p to regulate ZEB1 expression. Mol Cancer. 2018;17(1):119.

17. Yang FQ, Zhang JQ, Jin JJ, Yang CY, Zhang WJ, Zhang HM, et al. HOXA11-AS promotes the growth and invasion of renal cancer by sponging miR-146b-5p to upregulate MMP16 expression. J Cell Physiol. 2018;233(12):9611-9.

18. Gao H, Li X, Zhan G, Zhu Y, Yu J, Wang J, et al. Long noncoding RNA MAGl1-IT1 promoted invasion and metastasis of epithelial ovarian Cancer via the miR-200a/ZEB axis. Cell Cycle. 2019;18(12):1393-06.

19. Sun CC, Li SJ, Li G, Hua RX, Zhou XH, Li DJ. Long Intergenic noncoding RNA 00511 acts as an oncogene in non-smallcell lung cancer by binding to EZH2 and suppressing p57. Mol Ther Nucleic Acids. 2016;5(11):e385.

20. Lu G, Li Y, Ma Y, Lu J, Chen Y, Jiang $Q$, et al. Long noncoding RNA LINC00511 contributes to breast cancer tumourigenesis and stemness by inducing the miR-185-3p/E2F1/Nanog axis. J Exp Clin Cancer Res. 2018;37(1):289.

21. Kang YH, Lee SJ. The role of p38 MAPK and JNK in arsenic trioxide- induced mitochondrial cell death in human cervical cancer cells. J Cell Physiol. 2008;217(1):23-33.

22. Chen HJ, Lin CM, Lee CY, Shih NC, Peng SF, Tsuzuki M, et al. Kaempferol suppresses cell metastasis via inhibition of the ERK-p38-JNK and AP-1 signaling pathways in U-2 OS human osteosarcoma cells. Oncol Rep. 2013;30(2):925-32.

23. Song B, Li R, Zuo Z, Tan J, Liu L, Ding D, et al. LncRNA ENST00000539653 acts as an oncogenic factor via MAPK signalling in papillary thyroid cancer. BMC Cancer. 2019;19(1):297.

24. Yang MH, Zhao L, Wang L, Ou-Yang W, Hu SS, Li WL, et al. Nuclear IncRNA HOXD-AS1 suppresses colorectal carcinoma growth and metastasis via inhibiting HOXD3-induced integrin beta3 transcriptional activating and MAPK AKT signalling. Mol Cancer. 2019;18(1):31

25. Liu F, Tai $Y$, Ma J. LncRNA NEAT1/let-7a-5p axis regulates the cisplatin resistance in nasopharyngeal carcinoma by targeting Rsf-1 and modulating the Ras-MAPK pathway. Cancer Biol Ther. 2018;19(6):534-42.

26. Kataoka M, Wang DZ. Non-coding RNAs including miRNAs and IncRNAs in cardiovascular biology and disease. Cells. 2014;3(3):883-98

27. Chen $\mathrm{P}$, Wang R, Yue Q, Hao M. Long non-coding RNA TTN-AS1 promotes cell growth and metastasis in cervical cancer via miR-573/E2F3. Biochem Biophys Res Commun. 2018;503(4):2956-62.

28. Gao YS, Liu XZ, Zhang YG, Liu XJ, Li LZ. Knockdown of long noncoding RNA LUCAT1 inhibits cell viability and invasion by regulating miR-375 in Glioma. Oncol Res. 2018;26(2):307-13.

29. Zhang X, Zhou J, Xue D, Li Z, Liu Y, Dong L. MiR-515-5p acts as a tumor suppressor via targeting TRIP13 in prostate cancer. Int J Biol Macromol. 2019:129:227-32.

30. Li J, Tang Z, Wang H, Wu W, Zhou F, Ke H, et al. CXCL6 promotes non- small cell lung cancer cell survival and metastasis via down-regulation of miR-515-5p. Biomed Pharmacother. 2018:97:1182-8.

\section{Publisher's Note}

Springer Nature remains neutral with regard to jurisdictional claims in published maps and institutional affiliations. 